\title{
Bellman function, Littlewood-Paley estimates and asymptotics for the Ahlfors-Beurling operator in $L^{p}(\mathbb{C})$
}

\author{
Oliver Dragičević and Alexander Volberg
}

September 21, 2003

\section{Introduction}

Our goal is to present some estimates of the Ahlfors-Beurling operator and related plane Fourier multipliers. The Ahlfors-Beurling operator $T$ itself can be presented as a Fourier multiplier:

$$
(T f)^{\wedge}\left(\xi_{1}, \xi_{2}\right)=\frac{\left(\xi_{1}+i \xi_{2}\right)^{2}}{\xi_{1}^{2}+\xi_{2}^{2}} \hat{f}\left(\xi_{1}, \xi_{2}\right) .
$$

Estimation of $L^{p}$ norms of Fourier multipliers is known to be hard. It is usually connected to some interesting types of PDE, see several such PDE for several Fourier multipliers on the line in a recent paper of Kalton and Verbitsky [13]. Sometimes, but much more rarely, one can establish sharp $L^{p}$ estimates for Fourier multipliers in several variables. Riesz transforms are examples of success. They are defined in the following way. Choose $k \in\{1, \ldots, n\}$. The scalar Riesz transform $R_{k}$ acts on a test function $f$ (say, belonging to $C_{c}^{\infty}$ or the Schwartz class $\mathcal{S}$ ) by

$$
\left(R_{k} f\right)^{\wedge}(x)=i \frac{x_{k}}{\|x\|} \hat{f}(x) .
$$

For arbitrary functions $f \in L^{p}=L^{p}\left(\mathbb{R}^{n}\right)$ we extend this by density.

Pichorides [17] found an estimate from above for $\left\|R_{i}\right\|_{L^{p}\left(\mathbb{R}^{n}\right) \rightarrow L^{p}\left(\mathbb{R}^{n}\right)}$. The fact that this is actually also its estimate from below is proved in the paper of T. Iwaniec and G. Martin [12]. In the same paper the authors estimate the 
norm $\|\mathcal{R}\|_{L^{p}\left(\mathbb{R}^{n}\right) \rightarrow L^{p}\left(\mathbb{R}^{n}, l_{n}^{2}\right)}$ of vector Riesz transform $\mathcal{R}:=\left(R_{1}, \ldots, R_{n}\right)$. The same is done for $\mathcal{R}^{2}:=\left(R_{1}^{2}, \ldots, R_{n}^{2}\right)$. In this paper we will give some new estimates for $\|T\|_{p}:=\|T\|_{L^{p}\left(\mathbb{R}^{2}\right) \rightarrow L^{p}\left(\mathbb{R}^{2}\right)}$, whereas the new estimates of $\mathcal{R}^{2}$ from $L^{p}\left(\mathbb{R}^{n}\right)$ into $L^{p}\left(\mathbb{R}^{n}, l_{n}^{2}\right)$ will be proven elsewhere.

Throughout the paper, $p$ will be a number from $(1, \infty)$, while $q$ will stand for its conjugate exponent. Let us denote $p^{*}=\max \{p, q\}$. We will most often encounter the factor

$$
p^{*}-1=\left\{\begin{array}{llr}
p-1 & ; \quad & p \geq 2 \\
(p-1)^{-1} & ; \quad 1<p \leq 2
\end{array} .\right.
$$

The operator $\mathcal{R}$ can be naturally considered on $L^{p}\left(\mathbb{R}^{n}, l_{m}^{2}\right)$ as

$$
\left(R_{1}, \ldots, R_{n}\right)\left(f_{1}, \ldots, f_{m}\right):=\left[R_{i} f_{j}\right]_{\substack{i=1, ., n \\ j=1, \ldots, m}} .
$$

The estimate

$$
\left\|\mathcal{R}^{s}\right\|_{L^{p}\left(\mathbb{R}^{n}, l_{m}^{2}\right) \rightarrow L^{p}\left(\mathbb{R}^{n}, l_{m n}^{2}\right)} \leq 2\left(p^{*}-1\right) .
$$

was in the case of $s=1$ (i.e. when $\mathcal{R}^{s}=\mathcal{R}$ ) proven in [9], while the result for $s=2$ will follow elsewhere.

These results can be compared with Theorem 1.5 of [12], which says that one can replace $2\left(p^{*}-1\right)$ in $(1.1)$ by $\sqrt{2}^{s} H_{p}(s)$. Here $H_{p}(s):=\left\|\left(R_{1}+i R_{2}\right)^{s}\right\|_{p}$, where $R_{1}$ and $R_{2}$ are planar Riesz transforms. Notice that

$$
H_{p}(2)=\|T\|_{p}
$$

To our knowledge, neither $H_{p}(1)$ nor $H_{p}(2)$ are known. However, S.K. Pichorides proved that

$$
\left\|R_{k}\right\|_{p} \leq \cot \left(\frac{\pi}{2 p}\right) \quad \text { for } p \geq 2,
$$

where $R_{k}$ is a scalar Riesz transform on arbitrary $\mathbb{R}^{n}$. In particular, it immediately follows that

$$
H_{p}(1) \leq 2 \cot \left(\frac{\pi}{2 p}\right)
$$

for $p \geq 2$. Consequently

$$
\limsup _{p \rightarrow \infty} \frac{\sqrt{2} H_{p}(1)}{2(p-1)} \leq \frac{2 \sqrt{2}}{\pi}<1 .
$$


Thus for $m=1$ and $s=1$ the estimate (1.1) is weaker than the one in [12], provided that $p$ is large enough.

On the other hand, when $s=2$ the estimate in [12] prescribes $2 H_{p}(2)$. Our estimate (1.1) gives just the numerical estimate $2\left(p^{*}-1\right)$. It is conjectured, though, that

$$
H_{p}(2)=p-1 \quad \text { for } \quad p \geq 2 .
$$

In other words, we do not know how to derive (1.1) from the known results, it seems to be new for the case $s=2$.

As said before, the operator $T$ is given in the Fourier domain $\left(\xi_{1}, \xi_{2}\right)$ by the multiplier $[12$, p. 34]

$$
\frac{\zeta}{\bar{\zeta}}=\frac{\zeta^{2}}{|\zeta|^{2}}=\frac{\left(\xi_{1}+i \xi_{2}\right)^{2}}{\xi_{1}^{2}+\xi_{2}^{2}}=\frac{\xi_{1}^{2}}{\xi_{1}^{2}+\xi_{2}^{2}}-\frac{\xi_{2}^{2}}{\xi_{1}^{2}+\xi_{2}^{2}}+2 i \frac{\xi_{1} \xi_{2}}{\xi_{1}^{2}+\xi_{2}^{2}} .
$$

Thus, $T$ can be written as $T=R_{2}^{2}-R_{1}^{2}-2 i R_{1} R_{2}$, where $R_{1}, R_{2}$ are Riesz transforms on the plane. Another way of writing $T$ is

$$
T=m_{1}+i m_{2},
$$

where $m_{1}, m_{2}$ are Fourier multiplier operators. Notice that the multipliers themselves (as functions, not as multiplier operators) are connected by

$$
m_{2}=m_{1} \circ \rho,
$$

where $\rho(x, y)=\sqrt{2}^{-1}(y+x, y-x)$ is the clockwise $\pi / 4$ rotation of the plane. So the multiplier operators are related by

$$
m_{2}=U_{\rho} m_{1} U_{\rho}^{-1},
$$

where $U_{\rho} f=f \circ \rho$. Consequently, $m_{1}$ and $m_{2}$ admit the same $L^{p}$ estimates.

Let $L_{\text {real }}^{p}(d A)$ denote the $L^{p}$ space of real-valued functions with respect to the Lebesgue measure on the plane. We want the estimate

$$
\|T\|_{L_{r}^{p}(d A) \rightarrow L^{p}(d A)} \leq \tau(p) p, \text { where } \lim _{p \rightarrow \infty} \tau(p)=1 .
$$

The following asymptotic estimate on complex function will be also obtained:

$$
\|T\|_{L^{p}(d A) \rightarrow L^{p}(d A)} \leq \sqrt{2} \tau(p) p, \text { where } \lim _{p \rightarrow \infty} \tau(p)=1 .
$$


These both estimates will follow from the next one (valid for any $\vartheta \in$ $[0,2 \pi)$, and $p \geq 2)$

$$
\left\|\left(R_{1}^{2}-R_{2}^{2}\right) \cos \vartheta+2 R_{1} R_{2} \sin \vartheta\right\|_{L^{p}(d A) \rightarrow L^{p}(d A)} \leq p-1 .
$$

We discuss this inequality a bit further.

\section{The main estimate}

The key to our results will be this Littlewood-Paley-type inequality:

$$
\begin{aligned}
2 \int_{0}^{\infty} \int_{\mathbb{R}^{2}}\left(\left|\frac{\partial \tilde{f}}{\partial x_{1}}\right|^{2}\right. & \left.+\left|\frac{\partial \tilde{f}}{\partial x_{2}}\right|^{2}\right)^{1 / 2}\left(\left|\frac{\partial \tilde{g}}{\partial x_{1}}\right|^{2}+\left|\frac{\partial \tilde{g}}{\partial x_{2}}\right|^{2}\right)^{1 / 2} d y d t \\
& \leq\left(p^{*}-1\right)\|f\|_{p}\|g\|_{q},
\end{aligned}
$$

where $\tilde{f}$ and $\tilde{g}$ denote the heat extensions of $f$ and $g$, respectively.

Let us mention few things. First of all, the estimate (1.5) for $\vartheta=0$ was proved in [15]. This immediately yielded

$$
\|T\|_{L^{p}(d A) \rightarrow L^{p}(d A)} \leq 2(p-1)
$$

Estimate (1.6) is also proved by a different method in [3]. The previous best estimate was $4(p-1)$, see [4]. Here we will show how to win $\sqrt{2}$. This is based on a self-improvement of quadratic forms, as formulated in Lemma 3. At last, let us notice that getting rid of 2 in (1.6) entirely would yield very interesting geometric consequences. However, this seems to be very difficult. See [2], [3], [12], [15]. In [2] it is shown that " $p-1$ " - problem is very closely related to the famous Morrey's problem concerning quasiconvexity.

It would be interesting to get rid of 2 in (1.6) at least asymptotically, when $p \rightarrow \infty$. This might help to prove that the weak $1-1$ norm of $T$ is equal to 1 , which would have interesting geometric implications.

\subsection{Consequences of the " $p-1$ estimate"}

Let us formulate the analytic and geometric consequences of getting rid of 2 in (1.6). We are dealing with (local) solutions of the Beltrami equation 


$$
f_{\bar{z}}-\mu f_{z}=0 .
$$

We ask two questions:

1. Given $\|\mu\|_{\infty}=k<1$ and given that a solution is a priori in $W_{1}^{2}$ locally, what is the ensured smoothness of this local solution? It is classical that $f$ must belong to $W_{1}^{2+\varepsilon(k)}$ locally, where $\varepsilon(k)>0$. The best $\varepsilon(k)$ was the essence of the problem by F. Gehring solved by K. Astala [1]. The answer turned out to be equal to $\frac{1-k}{k}$. It is not attainable in general.

2. Given $\|\mu\|_{\infty}=k<1$ and given that solution is a priori in $W_{1}^{q}$ locally (now $q<2$ ), what is smallest $q$ which ensures smoothness $f \in W_{1}^{2}$ locally (and then by [1] ensures $f \in W_{1}^{1+1 / k-\tau}$ for any positive $\tau$ )? The smallest $q$ turns out to be $1+k$. And it is attainable (see [16]).

These both questions are intimately connected to estimate (1.6). Let us show why.

Consider a $W_{1}^{2}$ solution to (1.7) in a neighborhood $W$ of the origin. Let $V=\frac{1}{2} W$. Let $\varphi$ be a smooth function with image in $[0,1]$, supported in $W$ and identically equal to 1 on $V$. Put $g:=\varphi f_{\bar{z}}$. The assumption on $f$ implies that $g \in L^{2}$. Generalized $\bar{\partial}$ of the expression $-\frac{1}{\pi} \int_{\mathbb{C}} \frac{g(\zeta)}{z-\zeta} d A(\zeta)$ vanishes in $V$. So we have a function $h$ analytic in $V$, such that $f=h+\frac{1}{\pi} \int_{\mathbb{C}} \frac{g(\zeta)}{z-\zeta} d A(\zeta)$. So

$$
f_{z}=h^{\prime}-T g .
$$

in $V$. If we multiply (1.7) by $\varphi$ we thus get in $V$

$$
g-\mu T g=\mu h^{\prime}:=r .
$$

The function $r$ is bounded in $U:=\frac{1}{2} V$ and so belongs to any $L^{p}(U, d A)$. Let the operator of multiplication by $\mu$ in $L^{p}(U, d A)$ be called $M$. Clearly $\|M\| \leq k$. So if we denote $t(p):=\|T\|_{L^{p}(U, d A) \rightarrow L^{p}(U, d A)}$ and look at

$$
(I-M T) g=r,
$$

we see that from

$$
k t(p)<1
$$

it follows that $g \in L^{p}$ in $U$, which is the same as to say that $f \in W_{1}^{p}$ locally (because $g=f_{\bar{z}}$ on $V$ ). 
We see now that (1.6) without 2 would imply that for any $p$ such that

$$
p<1+\frac{1}{k}
$$

the solution of (1.7) which locally lies in $W_{1}^{2}$ also belongs to $L^{p}(d A)$ locally.

We can extract from this consideration the lower estimate on the norm of $T$ in $L^{p}$, namely $p-1$. In fact, if $t(p)$ would be strictly less than $p-1$ then (1.8) would show that if $\|\mu\|_{\infty}=k<1$ we have every solution (which is a priori in $W_{1}^{2}$ locally) of any $(1.7)$ with such a $\mu$ in $W_{1}^{1+1 / k+\varepsilon}$. But let us consider $f(z):=z|z|^{-\frac{2 k}{1+k}}$. It is easy to compute $\mu(z)=-k \frac{z}{\bar{z}}$. So its norm is $k$. However, $f$ is not in $W_{1}^{1+1 / k+\varepsilon}$ near the origin. It is not even in $W_{1}^{1+1 / k}$. In fact, it is readily computable that $\left|f_{z}\right|=C(k)|z|^{-\frac{2 k}{1+k}}$. And this does not belong to any $L^{p}(d A)$ for $p$ greater or equal to $1+1 / k$.

There is motivation enough to justify our interest in the estimation of the $L^{p}$-norms of such particular Fourier multipliers as $T$ and the multipliers related to $T$, which are being considered here.

\section{Heat extensions}

For $\varphi$ on the plane, its heat extension to the upper half-space $\mathbb{R}_{+}^{3}:=\mathbb{R}^{2} \times$ $(0, \infty)$ is given by the formula

$$
\widetilde{\varphi}(y, t):=\frac{1}{4 \pi t} \int_{\mathbb{R}^{2}} \varphi(x) e^{-\frac{|x-y|^{2}}{4 t}} d x ; \quad(y, t) \in \mathbb{R}_{+}^{3} .
$$

Such function solves the heat equation

$$
\Delta u=\frac{\partial u}{\partial t}
$$

in $\mathbb{R}_{+}^{3}$, whereas on the boundary $\mathbb{R}^{2} \times\{0\}$ it coincides with $\varphi$.

If $\varphi_{t}(y):=\widetilde{\varphi}(y, t)$, then obviously $\varphi_{t}=h_{t} * \varphi$ (the convolution being taken with respect to the measure $\frac{d m}{2 \pi}$ ), where

$$
h_{t}(x)=\frac{1}{2 t} e^{-\frac{\|x\|^{2}}{4 t}}, \quad x \in \mathbb{R}^{2}
$$

therefore $\widehat{\varphi}_{t}=\widehat{h_{t}} \widehat{\varphi}$. Furthermore, 


$$
\widehat{h}_{t}(x)=\frac{1}{2 \pi} \int_{\mathbb{R}^{2}} h_{t}(y) e^{-i\langle x, y\rangle} d y=e^{-t\|x\|^{2}} .
$$

From now on we will often use the same letter to denote both the function and its heat extension.

Lemma 1. Let $f, g \in C_{0}^{\infty}\left(\mathbb{R}^{2}\right)$ and $i, j \in\{1,2\}$. Then

$$
\iint_{\mathbb{R}^{2}} R_{i} R_{j} f \cdot g d x_{1} d x_{2}=-2 \int_{0}^{\infty} \int_{\mathbb{R}^{2}} \frac{\partial \widetilde{f}}{\partial x_{i}}(y, t) \frac{\partial \widetilde{g}}{\partial x_{j}}(y, t) d y d t
$$

or

$$
\left\langle R_{i} R_{j} f, g\right\rangle_{L^{2}\left(\mathbb{R}^{2}\right)}=-2\left\langle\frac{\partial \widetilde{f}}{\partial x_{i}}, \frac{\partial \widetilde{g}}{\partial x_{j}}\right\rangle_{L^{2}\left(\mathbb{R}_{+}^{3}\right)} .
$$

Proof. Take $f, g \in C_{0}^{\infty}\left(\mathbb{R}^{2}\right)$ and define $\eta:=g \circ\left(-\operatorname{id}_{\mathbb{R}^{2}}\right)$. Then

$$
\begin{aligned}
& \iint_{\mathbb{R}^{2}} R_{i} R_{j} f \cdot g d x_{1} d x_{2}=\left\langle R_{i} R_{j} f, \bar{g}\right\rangle=\left\langle\left(R_{i} R_{j} f\right)^{\wedge}, \hat{\bar{g}}\right\rangle=\left\langle\left(R_{i} R_{j} f\right)^{\wedge}, \overline{\hat{\eta}}\right\rangle \\
& =\iint_{\mathbb{R}^{2}}\left(R_{i} R_{j} f\right)^{\wedge} \cdot \hat{\eta} d x_{1} d x_{2}=-\iint_{\mathbb{R}^{2}} \frac{\xi_{i} \xi_{j}}{\xi_{1}^{2}+\xi_{2}^{2}} \hat{f}\left(\xi_{1}, \xi_{2}\right) \hat{\eta}\left(\xi_{1}, \xi_{2}\right) d \xi_{1} d \xi_{2} \\
& =-2 \iint_{\mathbb{R}^{2}} \xi_{i} \xi_{j} \int_{0}^{\infty} e^{-2 t\left(\xi_{1}^{2}+\xi_{2}^{2}\right)} d t \hat{f}\left(\xi_{1}, \xi_{2}\right) \hat{\eta}\left(\xi_{1}, \xi_{2}\right) d \xi_{1} d \xi_{2} \\
& =2 \int_{0}^{\infty} \iint_{\mathbb{R}^{2}} i \xi_{i} \hat{f}\left(\xi_{1}, \xi_{2}\right) e^{-t\left(\xi_{1}^{2}+\xi_{2}^{2}\right)} \cdot i \xi_{j} \hat{\eta}\left(\xi_{1}, \xi_{2}\right) e^{-t\left(\xi_{1}^{2}+\xi_{2}^{2}\right)} d \xi_{1} d \xi_{2} d t \\
& =2 \int_{0}^{\infty} \iint_{\mathbb{R}^{2}} \frac{\widehat{\partial f}}{\partial \xi_{i}}\left(\xi_{1}, \xi_{2}\right) e^{-t\left(\xi_{1}^{2}+\xi_{2}^{2}\right)} \cdot \frac{\widehat{\partial \eta}}{\partial \xi_{j}}\left(\xi_{1}, \xi_{2}\right) e^{-t\left(\xi_{1}^{2}+\xi_{2}^{2}\right)} d \xi_{1} d \xi_{2} d t .
\end{aligned}
$$

Using the facts listed just before the formulation of the Lemma, together with the Parseval equality, after some computation we see that this integral equals to

$$
-2 \int_{0}^{\infty} \int_{\mathbb{R}^{2}} \frac{\widetilde{\partial f}}{\partial x_{i}}(y, t) \frac{\widetilde{\partial g}}{\partial x_{j}}(y, t) d y d t
$$

and furthermore to

$$
-2 \int_{0}^{\infty} \int_{\mathbb{R}^{2}} \frac{\partial \widetilde{f}}{\partial x_{i}}(y, t) \frac{\partial \widetilde{g}}{\partial x_{j}}(y, t) d y d t
$$


which had to be proven.

Our next goal is to estimate the right side of (1.9) from above. The following theorem was proved in [15].

Theorem 1. For any $f, g \in C_{0}^{\infty}\left(\mathbb{R}^{2}\right)$ and $i, j \in\{1,2\}$ we have

$$
\begin{gathered}
2 \int_{0}^{\infty} \int_{\mathbb{R}^{2}}\left|\frac{\partial f}{\partial x_{1}}\right|\left|\frac{\partial g}{\partial x_{1}}\right| d x d t+2 \int_{0}^{\infty} \int_{\mathbb{R}^{2}}\left|\frac{\partial f}{\partial x_{2}}\right|\left|\frac{\partial g}{\partial x_{2}}\right| d x d t \\
\leq\left(p^{*}-1\right)\|f\|_{p}\|g\|_{q} .
\end{gathered}
$$

Corollary 1. In particular,

$$
\left\|R_{1}^{2}-R_{2}^{2}\right\|_{p} \leq p^{*}-1, \quad\left\|2 R_{1} R_{2}\right\|_{p} \leq p^{*}-1 .
$$

This corollary immediately implies (1.6). But to obtain (1.3), (1.4) and (1.5) we need a stronger estimate. It will be obtained by the use of the same technique as in the proof of Theorem 1, but also we need to apply Lemma 3. Here is a strengthened version of Theorem 1. This is our new Littlewood-Paley inequality for the heat kernel.

Theorem 2. For any $f, g \in C_{0}^{\infty}\left(\mathbb{R}^{2}\right)$ we have

$$
\begin{aligned}
2 \int_{0}^{\infty} \int_{\mathbb{R}^{2}}\left(\left|\frac{\partial f}{\partial x_{1}}\right|^{2}\right. & \left.+\left|\frac{\partial f}{\partial x_{2}}\right|^{2}\right)^{1 / 2}\left(\left|\frac{\partial g}{\partial x_{1}}\right|^{2}+\left|\frac{\partial g}{\partial x_{2}}\right|^{2}\right)^{1 / 2} d x d t \\
& \leq\left(p^{*}-1\right)\|f\|_{p}\|g\|_{q} .
\end{aligned}
$$

The following corollary from Theorem 2 proves (1.5). In its turn, (1.5) proves, as we will see, (1.3) and (1.4).

\section{Corollary 2.}

$$
\left\|\left(R_{1}^{2}-R_{2}^{2}\right) \cos \vartheta+2 R_{1} R_{2} \sin \vartheta\right\|_{p} \leq p^{*}-1 .
$$

Both corollaries (but not the theorems) above were proven by R. Bañuelos and P. Méndez-Hernández in [3]. Their method, however, is different.

Before proceeding with the proofs, let us describe the most important ingredient of our approach. It has already been presented in [15]. In order to keep this paper self-contained, we repeat the corresponding part of [15]. 


\section{The Bellman function}

Take $p \in(1, \infty)$ and assume that $M$ and $N$ are natural numbers. Define

$$
\Omega=\left\{(\zeta, \eta, Z, H) \in \mathbb{C}^{M} \times \mathbb{C}^{N} \times \mathbb{R} \times \mathbb{R} ;|\zeta|^{p}<Z,|\eta|^{q}<H\right\} .
$$

This is a convex domain in $\mathbb{C}^{M} \times \mathbb{C}^{N} \times \mathbb{R} \times \mathbb{R} \equiv \mathbb{R}^{d}, d=2(M+N+1)$.

Theorem 3. There is a function $B: \bar{\Omega} \rightarrow \mathbb{R}$, such that

1.

$$
0 \leq B(\zeta, \eta, Z, H) \leq\left(p^{*}-1\right) Z^{1 / p} H^{1 / q}
$$

everywhere on its domain;

2.

$$
B\left(\frac{a_{+}+a_{-}}{2}\right)-\frac{B\left(a_{+}\right)+B\left(a_{-}\right)}{2} \geq\left|\frac{\zeta_{+}-\zeta_{-}}{2}\right| \cdot\left|\frac{\eta_{+}-\eta_{-}}{2}\right|
$$

for any $a_{ \pm}=\left(\zeta_{ \pm}, \eta_{ \pm}, Z_{ \pm}, H_{ \pm}\right) \in \bar{\Omega}$.

If $K$ is a compact subset of $\Omega$ and $\kappa$ is a positive number, smaller than $\min \{1, d(K, \partial \Omega)\}$, then there exists a smooth function $B_{K, \kappa}$ on the neighbourhood $\Omega_{\kappa}:=\{x \in \Omega ; d(x, \partial \Omega)>\kappa\}$ of $K$, such that the second estimate still holds. In that case, it takes the form

2'.

$$
-d^{2} B_{K}(\zeta, \eta, Z, H) \geq 2|d \zeta||d \eta| .
$$

The first estimate is perturbed only by the factor $1+\kappa$.

We will deliver the proof of this theorem in Section 5 .

This is our Bellman function. By the second property we mean that for any choice of $(\zeta, \eta, Z, H) \in \Omega$ and $(\alpha, \beta, \gamma, \delta) \in \mathbb{C}^{M} \times \mathbb{C}^{N} \times \mathbb{R} \times \mathbb{R}$ we have

$$
\left\langle-d^{2} B(\zeta, \eta, Z, H)[\alpha, \beta, \gamma, \delta]^{T},[\alpha, \beta, \gamma, \delta]^{T}\right\rangle \geq 2|\alpha||\beta| .
$$

Naturally, the Hessian $d^{2} B$ can be thought of as a real $d \times d$ matrix, whose upper left $2 \times 2$ submatrix, for instance, equals 


$$
\left[\frac{\partial^{2} B}{\partial \zeta_{i}^{(1)} \partial \zeta_{j}^{(1)}}\right]_{i, j=1,2}
$$

where $\zeta=\left(\zeta^{(1)}, \ldots, \zeta^{(M)}\right)$ and $\zeta^{(1)}=\zeta_{1}^{(1)}+i \zeta_{2}^{(1)}$.

In general we do not know of a formula, i.e. an algebraic expression, which would possess the properties of a Bellman function. The only exception occurs when $p=2$. In that case one possible choice for $B$ is given by

$$
B(\zeta, \eta, Z, H)=\sqrt{\left(Z-|\zeta|^{2}\right)\left(H-|\eta|^{2}\right)} .
$$

\section{Proof of Theorem 2.}

We shall invoke the Bellman function that was announced in Theorem 3. We will use only the special case when $M=N=1$. Choose $p \in(1, \infty)$ and two test functions $f, g \in C_{0}^{\infty}\left(\mathbb{R}^{2}\right)$. Define, for $(x, t) \in \mathbb{R}^{2} \times(0, \infty)$,

$$
v(x, t):=\left(f(x, t), g(x, t),|f|^{p}(x, t),|g|^{q}(x, t)\right)
$$

We emphasize that the components of $v$ are in fact the heat extensions of functions $f, g,|f|^{p}$ and $|g|^{q}$, respectively. Having this in mind, we see that $v$ maps into the region $\Omega$, defined in Theorem 3 .

Next, we introduce a Green's function $G(x, t)$ as in [11]. Fix $l>1$ and consider the cylinder $Q=Q_{l}:=B(0, l) \times(0, l)$. Denote $\partial^{\prime} Q=\partial B(0, l) \times(0, l)$. These are the properties of our Green's function:

$$
\left\{\begin{aligned}
\left(\frac{\partial}{\partial t}+\Delta\right) G_{Q} & =-\delta_{0,1} & & \text { in } Q \\
G_{Q} & =0 & & \text { on } \partial^{\prime} Q \\
G_{Q} & =0 & & \text { when } t=l .
\end{aligned}\right.
$$

Here $\delta_{0,1}$ is the delta-function of the point $(0,1)$.

Let $k(x, t):=\frac{1}{4 \pi t} e^{-\frac{|x|^{2}}{4 t}},(x, t) \in \mathbb{R}^{2} \times(0, \infty)$ be a heat kernel in $\mathbb{R}_{+}^{3}$. One can understand $k(0, t)$ as the temperature of point $(0,0)$ on the plain at time $t>0$, if initially (at $t=0$ ) one has the delta distribution of temperature placed at $(0,0)$. It is important to keep in mind that

$$
G_{Q}(0,0) \rightarrow k(0,1) \text {, when } l \rightarrow \infty \text {. }
$$


In fact, just compare the interpretation of $k(0,1)$ with the fact that $G_{Q}(0,0)$ is temperature at time 1 when the same initial distribution is given but when also the temperature on $\partial^{\prime} Q$ is kept to be 0 . However, when $l$ is large, it is clear that these two quantities are very close.

For $R>0$ we also need a Green's function in the cylinder $Q\left(R, R^{2}\right):=$ $B(0, l R) \times\left(0, l R^{2}\right):$

$$
\left\{\begin{array}{rlrl}
\left(\frac{\partial}{\partial t}+\Delta\right) G_{Q}^{R} & =-\delta_{0, R^{2}} & & \text { in } Q\left(R, R^{2}\right)=B(0, l R) \times\left(0, l R^{2}\right), \\
G_{Q}^{R} & =0 & & \text { on } \partial^{\prime} Q\left(R, R^{2}\right)=\partial B(0, l R) \times\left(0, l R^{2}\right), \\
G_{Q}^{R}=0 & & \text { when } t=l R^{2} .
\end{array}\right.
$$

In addition, choose $0<\delta<1$ and denote $Q_{R, \delta}:=Q\left(R, R^{2}\right) \cap\{t>\delta\}$. Take an arbitrary compact set $M \subset \mathbb{R}^{2} \times(\delta, \infty)$. Fix also some $l>1$. There is $R>0$ such that $M \subset Q_{R, \delta}$. The set $K:=v\left(\overline{Q_{R, \delta}}\right)$ is compact and contained in $\Omega$. Let $0<\kappa<\min \left\{1, R^{-1}\right\}$ be small enough for $d(K, \partial \Omega) \geq \kappa$. Recall the notation $\Omega_{\kappa}:=\{a \in \Omega ; d(a, \partial \Omega)>\kappa\}$. If $U:=v^{-1}\left(\Omega_{\kappa}\right)$, then the function $b:=B_{K, \kappa} \circ v$ maps $U \rightarrow[0, \infty)$. In particular, it is defined on $Q_{R, \delta}$.

For such chosen $R$ we apply the Green's formula in $Q\left(R, R^{2}\right)$.

$$
\begin{aligned}
b\left(0, R^{2}\right)= & -\iiint_{Q_{R, \delta}}\left(\frac{\partial}{\partial t}+\Delta\right) G_{Q}^{R}(x, t) b(x, t) d x_{1} d x_{2} d t \\
= & \iiint_{Q_{R, \delta}} G_{Q}^{R}(x, t)\left(\frac{\partial}{\partial t}-\Delta\right) b(x, t) d x_{1} d x_{2} d t \\
& +\iint_{B(0, R)} b(x, \delta) G_{Q}^{R}(x, \delta) d x_{1} d x_{2} \\
& +\iint_{\partial^{\prime} Q\left(R, R^{2}\right) \cap\{t>\delta\}}\left(\frac{\partial b}{\partial n_{\text {outer }}} G_{Q}^{R}-\frac{\partial G_{Q}^{R}}{\partial n_{\text {outer }}} b\right) d s d t \\
= & \iiint_{Q_{R, \delta}} G_{Q}^{R}(x, t)\left(\frac{\partial}{\partial t}-\Delta\right) b(x, t) d x_{1} d x_{2} d t \\
& +\iint_{B(0, l R)} b(x, \delta) G_{Q}^{R}(x, \delta) d x_{1} d x_{2} \\
& +\iint_{\partial^{\prime} Q\left(R, R^{2}\right) \cap\{t>\delta\}} \frac{\partial G_{Q}^{R}}{\partial n_{\text {inner }}} b d s d t
\end{aligned}
$$




$$
\geq \iiint_{Q_{R, \delta}} G_{Q}^{R}(x, t)\left(\frac{\partial}{\partial t}-\Delta\right) b(x, t) d x_{1} d x_{2} d t .
$$

The last inequality is clear: the double integrals are both nonnegative, because $b$ is nonnegative and because $G_{Q}^{R}$ is nonnegative and vanishes on the side boundary.

On the other hand, since $v\left(0, R^{2}\right) \in \Omega_{\kappa}$, we get from Theorem 3

$$
\begin{aligned}
b\left(0, R^{2}\right) & \leq(1+\kappa)\left(p^{*}-1\right)\left(|f|^{p}\left(0, R^{2}\right)\right)^{1 / p}\left(|g|^{q}\left(0, R^{2}\right)\right)^{1 / q}= \\
& =(1+\kappa)\left(p^{*}-1\right)\left(\frac{1}{4 \pi R^{2}} \int_{\mathbb{R}^{2}}|f(x)|^{p} e^{-\frac{|x|^{2}}{4 R^{2}}} d x\right)^{1 / p}\left(\ldots|g|^{q} \ldots\right)^{1 / q} \\
& \leq \frac{(1+\kappa)\left(p^{*}-1\right)}{4 \pi R^{2}}\|f\|_{p}\|g\|_{q} .
\end{aligned}
$$

The combination of recent estimates for $b\left(0, R^{2}\right)$ gives

$$
\iiint_{Q_{R, \delta}} G_{Q}^{R}(x, t)\left(\frac{\partial}{\partial t}-\Delta\right) b(x, t) \leq \frac{(1+\kappa)\left(p^{*}-1\right)}{4 \pi R^{2}}\|f\|_{p}\|g\|_{q} .
$$

At this point we need the two auxiliary results stated below. They allows us to apply the second key property of the Bellman function (see Theorem 3).

Lemma 2. If $(x, t) \in U$ then

$$
\left(\frac{\partial}{\partial t}-\Delta\right) b(x, t)=\left\langle\left(-d^{2} B\right) \frac{\partial v}{\partial x_{1}}, \frac{\partial v}{\partial x_{1}}\right\rangle_{\mathbb{R}^{6}}+\left\langle\left(-d^{2} B\right) \frac{\partial v}{\partial x_{2}}, \frac{\partial v}{\partial x_{2}}\right\rangle_{\mathbb{R}^{6}} .
$$

Proof. Choose $x_{0}=(x, t) \in M$. Denote also $v_{0}=v\left(x_{0}\right)$. Using the chain rule gives

$$
\frac{\partial b}{\partial x_{i}}\left(x_{0}\right)=\left\langle\nabla B\left(v_{0}\right), \frac{\partial v}{\partial x_{i}}\left(x_{0}\right)\right\rangle_{\mathbb{R}^{6}} ; \quad i=1,2
$$

and similarly for the partial derivative with respect to $t$, which implies

$$
\Delta b\left(x_{0}\right)=\left\langle\nabla B\left(v_{0}\right), \Delta v\left(x_{0}\right)\right\rangle+\sum_{i=1}^{2}\left\langle d^{2} B\left(v_{0}\right) \frac{\partial v}{\partial x_{i}}\left(x_{0}\right), \frac{\partial v}{\partial x_{i}}\left(x_{0}\right)\right\rangle .
$$

So far the formulæ are true for arbitrary $B, v, b=B \circ v, x_{0}, v_{0}=v\left(x_{0}\right)$.

Now we use that $v$ is comprised of heat extensions, which, after subtracting $\frac{\partial b}{\partial t}\left(x_{0}\right)$, forces the first term on the right to vanish. This finishes the proof of lema 2. 
Lemma 3. Let $m, n, k \in \mathbb{N}$. Denote $d=m+n+k$. For arbitrary $v \in \mathbb{R}^{m+n+k}$ write $v=v_{m} \oplus v_{n} \oplus v_{k}$, where $v_{i} \in \mathbb{R}^{i}$ for $i=m, n, k$. Let $R=\left\|v_{m}\right\|, r=\left\|v_{n}\right\|$. Suppose matrix $A \in \mathbb{R}^{d, d}$ is such that

$$
\langle A v, v\rangle \geq 2 R r
$$

for all $v \in \mathbb{R}^{d}$. Then there is $\tau>0$, satisfying

$$
\langle A v, v\rangle \geq \tau R^{2}+\frac{1}{\tau} r^{2}
$$

again for all $v \in \mathbb{R}^{d}$. In particular, the (restriction) operator $\left.A\right|_{\mathbb{R}^{m+n} \times\{0\}^{k}}$ "becomes" injective.

This lemma will be proven in the Addendum.

The two lemmas, combined with Theorem 3, imply that there is $\tau>0$ such that for all $(x, t) \in Q_{R, \delta}$,

$$
\begin{aligned}
\left(\frac{\partial}{\partial t}-\Delta\right) b(x, t) & \geq \tau\left|\frac{\partial f}{\partial x_{1}}\right|^{2}+\tau^{-1}\left|\frac{\partial g}{\partial x_{1}}\right|^{2} \\
& +\tau\left|\frac{\partial f}{\partial x_{2}}\right|^{2}+\tau^{-1}\left|\frac{\partial g}{\partial x_{2}}\right|^{2}
\end{aligned}
$$

and consequently

$$
\left(\frac{\partial}{\partial t}-\Delta\right) b(x, t) \geq 2\left(\left|\frac{\partial f}{\partial x_{1}}\right|^{2}+\left|\frac{\partial f}{\partial x_{2}}\right|^{2}\right)^{1 / 2}\left(\left|\frac{\partial g}{\partial x_{1}}\right|^{2}+\left|\frac{\partial g}{\partial x_{2}}\right|^{2}\right)^{1 / 2}
$$

Since the integrand in (3.2) is positive and $M \subset Q_{R, \delta}$, the previous line gives

$$
\begin{gathered}
2 \iiint_{M} G_{Q}^{R}(x, t)\left(\left|\frac{\partial f}{\partial x_{1}}\right|^{2}+\left|\frac{\partial f}{\partial x_{2}}\right|^{2}\right)^{1 / 2}\left(\left|\frac{\partial g}{\partial x_{1}}\right|^{2}+\left|\frac{\partial g}{\partial x_{2}}\right|^{2}\right)^{1 / 2} \\
\leq \frac{(1+\kappa)\left(p^{*}-1\right)}{4 \pi R^{2}}\|f\|_{p}\|g\|_{q}
\end{gathered}
$$

One can easily see that

$$
G_{Q}^{R}(x, t)=\frac{1}{R^{2}} G_{Q}\left(\frac{x}{R}, \frac{t}{R^{2}}\right)
$$


so (3.4) implies

$$
\begin{aligned}
2 \iiint_{M} G_{Q}\left(\frac{x}{R}, \frac{t}{R^{2}}\right) & \left(\left|\frac{\partial f}{\partial x_{1}}\right|^{2}+\left|\frac{\partial f}{\partial x_{2}}\right|^{2}\right)^{1 / 2}\left(\left|\frac{\partial g}{\partial x_{1}}\right|^{2}+\left|\frac{\partial g}{\partial x_{2}}\right|^{2}\right)^{1 / 2} \\
& \leq \frac{(1+\kappa)\left(p^{*}-1\right)}{4 \pi}\|f\|_{p}\|g\|_{q}
\end{aligned}
$$

for all $R>0$ large enough for $Q_{R, \delta}$ to contain $M$. Taking into account that

$$
\lim _{R \rightarrow \infty} G_{Q}\left(\frac{x}{R}, \frac{t}{R^{2}}\right)=G_{Q}(0,0)
$$

uniformly on the compact $M$ and that $\kappa<R^{-1}$ by construction, we obtain

$$
\begin{gathered}
2 G_{Q_{l}}(0,0) \iiint_{M}\left(\left|\frac{\partial f}{\partial x_{1}}\right|^{2}+\left|\frac{\partial f}{\partial x_{2}}\right|^{2}\right)^{1 / 2}\left(\left|\frac{\partial g}{\partial x_{1}}\right|^{2}+\left|\frac{\partial g}{\partial x_{2}}\right|^{2}\right)^{1 / 2} \\
\leq \frac{\left(p^{*}-1\right)}{4 \pi}\|f\|_{p}\|g\|_{q} .
\end{gathered}
$$

Now it is time to tend $Q=Q_{l}$ to $\mathbb{R}_{+}^{3}$ by making $l$ go to infinity. By (3.1) we conclude that $G_{Q_{l}}(0,0) \rightarrow \frac{1}{4 \pi}$. So (3.6) becomes

$$
2 \iiint_{M}\left(\left|\frac{\partial f}{\partial x_{1}}\right|^{2}+\left|\frac{\partial f}{\partial x_{2}}\right|^{2}\right)^{1 / 2}\left(\left|\frac{\partial g}{\partial x_{1}}\right|^{2}+\left|\frac{\partial g}{\partial x_{2}}\right|^{2}\right)^{1 / 2} \leq\left(p^{*}-1\right)\|f\|_{p}\|g\|_{q}
$$

Since $M$ was an arbitrary compact set in the upper half space, the Theorem 2 is proven.

\section{Applications of Theorem 2: asymptotic behavior of $\|T\|_{p}$}

We start by proving Corollary 2.

Proof. Fix two test functions $f, g \in C_{0}^{\infty}\left(\mathbb{R}^{2}\right)$. Let

$$
T_{\vartheta}:=\left(R_{1}^{2}-R_{2}^{2}\right) \cos \vartheta+2 R_{1} R_{2} \sin \vartheta .
$$


We can write using Lemma 1

$$
\left\langle T_{\vartheta} f, g\right\rangle=-2 \int_{\mathbb{R}_{+}^{3}}\left[\left(\frac{\partial f}{\partial x_{1}} \frac{\partial g}{\partial x_{1}}-\frac{\partial f}{\partial x_{2}} \frac{\partial g}{\partial x_{2}}\right) \cos \vartheta+2 \frac{\partial f}{\partial x_{1}} \frac{\partial g}{\partial x_{2}} \sin \vartheta\right] .
$$

The absolute value of the integrand is

$$
\begin{gathered}
\left|\left\langle\left(\begin{array}{cc}
\cos \vartheta & \sin \vartheta \\
\sin \vartheta & -\cos \vartheta
\end{array}\right)\left(\begin{array}{c}
\frac{\partial f}{\partial x_{1}} \\
\frac{\partial f}{\partial x_{2}}
\end{array}\right),\left(\begin{array}{c}
\frac{\partial g}{\partial x_{1}} \\
\frac{\partial g}{\partial x_{2}}
\end{array}\right)\right\rangle_{\mathbb{C}^{2}}\right| \\
\leq\left(\left|\frac{\partial f}{\partial x_{1}}\right|^{2}+\left|\frac{\partial f}{\partial x_{2}}\right|^{2}\right)^{1 / 2}\left(\left|\frac{\partial g}{\partial x_{1}}\right|^{2}+\left|\frac{\partial g}{\partial x_{2}}\right|^{2}\right)^{1 / 2} .
\end{gathered}
$$

Combining these two relations we get

$$
\left|\left\langle T_{\vartheta} f, g\right\rangle\right| \leq 2 \int_{\mathbb{R}_{+}^{3}}\left(\left|\frac{\partial f}{\partial x_{1}}\right|^{2}+\left|\frac{\partial f}{\partial x_{2}}\right|^{2}\right)^{1 / 2}\left(\left|\frac{\partial g}{\partial x_{1}}\right|^{2}+\left|\frac{\partial g}{\partial x_{2}}\right|^{2}\right)^{1 / 2} .
$$

The use of Theorem 2 now leads to Corollary 2 and (1.5).

Now we want to prove (1.3) and (1.4), which are certain asymptotic estimates for $\|T\|_{p}, p \rightarrow \infty$.

We need the following

Lemma 4. Let $\mu$ be a positive measure on space $X$ and let $A, B$ be operators acting on $L_{\text {real }}^{p}(X, \mu)$. Denote

$$
C(p):=\max _{\vartheta \in[0,2 \pi)}\|A \cos \vartheta+B \sin \vartheta\|_{p} .
$$

Then

$$
\left\|\left(\begin{array}{l}
A \\
B
\end{array}\right): L_{\text {real }}^{p} \rightarrow L^{p}\left(l_{\text {real }}^{2}\right)\right\| \leq \tau_{p} C(p), \text { where } \quad \lim _{p \rightarrow \infty} \tau_{p}=1 .
$$

Proof. It almost repeats the proof of the frequently used MarcinkiewiczZygmund lemma, which asserts that the operator acting on real $L^{p}$ acts on complex $L^{p}$ with the same norm. The trick in the proof of MarcinkiewiczZygmund lemma was to act on $f \cos \vartheta+g \sin \vartheta$.

Now instead of acting by $T$ on $f \cos \vartheta+g \sin \vartheta$ we fix $u \in L_{\text {real }}^{p}(\mu)$ and apply to it $A \cos \vartheta+B \sin \vartheta$. Take $\omega \in[0,2 \pi)$ and write temporarily $a=$ 
$A u(\omega), b=B u(\omega)$. Since $a$ and $b$ are real (by the assumption of the lemma), there is $\delta=\delta(\omega) \in[0,2 \pi)$ such that

$$
(a, b)=\sqrt{a^{2}+b^{2}}(\cos \delta, \sin \delta) \in \mathbb{R}^{2} .
$$

It follows that

$$
a \cos \vartheta+b \sin \vartheta=\sqrt{a^{2}+b^{2}} \cos (\vartheta-\delta)
$$

or, in other words,

$$
(A \cos \vartheta+B \sin \vartheta) u(\omega)=\left(|A u(\omega)|^{2}+|B u(\omega)|^{2}\right)^{1 / 2} \cos (\vartheta-\delta(\omega))
$$

for all $\vartheta \in[0,2 \pi)$. Consequently,

$$
\begin{aligned}
& \int_{X}\left(|A u(\omega)|^{2}+|B u(\omega)|^{2}\right)^{p / 2}|\cos (\vartheta-\delta(\omega))|^{p} d \mu(\omega) \\
= & \int_{X}|(A \cos \vartheta+B \sin \vartheta) u(\omega)|^{p} d \mu(\omega) \leq C(p)^{p}\|u\|_{p}^{p} .
\end{aligned}
$$

Integrate this inequality with respect to the normalized Lebesgue measure $(2 \pi)^{-1} d \vartheta$ on $[0,2 \pi)$. By denoting

$$
\tau_{p}:=\left(\frac{1}{2 \pi} \int_{0}^{2 \pi}|\cos \vartheta|^{p} d \vartheta\right)^{-1 / p}
$$

we get

$$
\left(\int\left(|A u(\omega)|^{2}+|B u(\omega)|^{2}\right)^{p / 2} d \mu(\omega)\right)^{1 / p} \leq \tau_{p} C(p)\|u\|_{p},
$$

and Lemma 4 is proved.

For $A=R_{1}^{2}-R_{2}^{2}$ and $B=2 R_{1} R_{2}$, this lemma and Corollary 2 give exactly (1.3). Thus we have, for real-valued $u$,

$$
\left(\int\left(\left|\left(R_{1}^{2}-R_{2}^{2}\right) u\right|^{2}+\left|2 R_{1} R_{2} u\right|^{2}\right)^{p / 2} d \mu\right)^{1 / p} \leq \tau_{p}(p-1)\|u\|_{p}, \quad \lim _{p \rightarrow \infty} \tau_{p}=1 .
$$

We are left to prove (1.4). It would follow immediately if the following variant of Marcinkiewicz-Zygmund lemma would hold. First let us mention that the classical Marcinkiewicz-Zygmund lemma states (all spaces are real): 
if $T: L^{p} \rightarrow L^{p}$ is bounded, then $T: L^{p}\left(l^{2}\right) \rightarrow L^{p}\left(l^{2}\right)$ is bounded, and has the same norm. However strange this may be, it seems to us that the following very close analogue of Marcinkiewicz-Zygmund lemma does not hold. Let again all spaces be real. Let $T: L^{p} \rightarrow L^{p}\left(l^{2}\right)$ be bounded. We would wish to state that then $T: L^{p}\left(l^{2}\right) \rightarrow L^{p}\left(l^{2} \otimes l^{2}\right)$ has the same norm. But this is false!

Suppose for the moment that such "tensorisation" were true. Then for real functions $u, v$ we have the analogue of (4.1) $\left(A=R_{1}^{2}-R_{2}^{2}, B=2 R_{1} R_{2}\right)$

$$
\begin{gathered}
{\left[\int\left(|A u|^{2}+|B u|^{2}+|A v|^{2}+|B v|^{2}\right)^{p / 2} d \mu\right]^{1 / p}} \\
\leq \tau_{p}(p-1)\|u+i v\|_{p}, \lim _{p \rightarrow \infty} \tau_{p}=1 .
\end{gathered}
$$

We are sure that (4.2) is correct. And of course it gives (1.4) because then obviously

$$
\left(\int|(A+i B)(u+i v)|^{p} d \mu\right)^{1 / p} \leq \sqrt{2} \tau_{p}(p-1)\|u+i v\|_{p}, \lim _{p \rightarrow \infty} \tau_{p}=1 .
$$

However, the "tensorisation" which led to (4.2) is false, as said before. So we have to prove (4.3) without using (4.2).

First we write $|(A+i B)(u+i v)|^{p}=\left(|A u-B v|^{2}+|B u+A v|^{2}\right)^{p / 2}$. We use the same trick as in the proof of Lemma 4 , namely, we introduce $\cos \vartheta, \sin \vartheta$ as follows. Considering a (real-valued) test function $\psi$,

$$
\langle(A u-B v) \cos \theta+(B u+A v) \sin \theta, \psi\rangle=\int_{\mathbb{R}^{2}}\left\langle\left(\begin{array}{rr}
A & -B \\
B & A
\end{array}\right)\left(\begin{array}{l}
u \\
v
\end{array}\right),\left(\begin{array}{l}
\psi \cos \vartheta \\
\psi \sin \vartheta
\end{array}\right)\right\rangle .
$$

It is easy to see that our last integral is just $\Re\left\langle(A+i B)(u+i v), e^{i \vartheta} \psi\right\rangle$. Use this for $A=R_{1}^{2}-R_{2}^{2}$ and $B=2 R_{1} R_{2}$ and rewrite the expression by using Lemma 1. We get

$$
\begin{gathered}
\langle(A u-B v) \cos \vartheta+(B u+A v) \sin \vartheta, \psi\rangle \\
=-2 \Re e^{-i \vartheta} \int_{\mathbb{R}_{+}^{3}}\left(\frac{\partial}{\partial x_{1}}+i \frac{\partial}{\partial x_{2}}\right)(u+i v)\left(\frac{\partial}{\partial x_{1}}+i \frac{\partial}{\partial x_{2}}\right) \psi .
\end{gathered}
$$

Of course here $u, v, \psi$ are heat extensions of our initial $u, v, \psi$. Let us fix $t>0$ and consider $J(u, v)$, the Jacobian of the map $(u(x, t), v(x, t)): \mathbb{R}^{2} \rightarrow \mathbb{R}^{2}$. 
Then our previous equality gives us

$$
\begin{gathered}
|\langle(A u-B v) \cos \vartheta+(B u+A v) \sin \vartheta, \psi\rangle| \\
\leq 2 \int_{\mathbb{R}_{+}^{3}}\left|\left(\frac{\partial}{\partial x_{1}}+i \frac{\partial}{\partial x_{2}}\right)(u+i v)\right| \cdot\left|\left(\frac{\partial}{\partial x_{1}}+i \frac{\partial}{\partial x_{2}}\right) \psi\right| \\
\leq 2 \int_{\mathbb{R}_{+}^{3}}\left(\left|\frac{\partial u}{\partial x_{1}}\right|^{2}+\left|\frac{\partial u}{\partial x_{2}}\right|^{2}+\left|\frac{\partial v}{\partial x_{1}}\right|^{2}+\left|\frac{\partial v}{\partial x_{2}}\right|^{2}-2 \operatorname{det} J(u, v)\right)^{1 / 2} \\
\leq 2 \int_{\mathbb{R}_{+}^{3}}\left(\left|\frac{\partial u}{\partial x_{1}}\right|^{2}+\left|\frac{\partial u}{\partial x_{2}}\right|^{2}+\left|\frac{\partial v}{\partial x_{1}}\right|^{2}+\left|\frac{\partial v}{\partial x_{2}}\right|^{2}\right)^{1 / 2} \cdot \\
=2 \sqrt{2} \int_{\mathbb{R}_{+}^{3}}\left(\left|\frac{\partial \psi}{\partial x_{1}}\right|^{2}+\left|\frac{\partial \psi}{\partial x_{1}}\right|^{2}+\left|\frac{\partial \psi}{\partial x_{2}}\right|^{2}\right)^{1 / 2}\left(\left|\frac{\partial \psi}{\partial x_{1}}\right|^{2}+\left|\frac{\partial \psi}{\partial x_{2}}\right|^{2}\right)^{1 / 2}
\end{gathered}
$$

where $\varphi:=u+i v$. Now we have to use Theorem 2 again. It gives that the last expression is bounded by $\sqrt{2}(p-1)\|u+i v\|_{p}\|\psi\|_{q}$. Gathering all together, one obtains

$$
|\langle(A u-B v) \cos \vartheta+(B u+A v) \sin \vartheta, \psi\rangle| \leq \sqrt{2}(p-1)\|u+i v\|_{p}\|\psi\|_{q} .
$$

And so

$$
\|\left\langle(A u-B v) \cos \vartheta+(B u+A v) \sin \vartheta\left\|_{p} \leq \sqrt{2}(p-1)\right\| u+i v \|_{p} .\right.
$$

Once again we utilize the trick used in the proof of Lemma 4:

$$
\begin{gathered}
(A u-B v) \cos \vartheta+(B u+A v) \sin \vartheta \\
=\left(|A u-B v|^{2}+|B u+A v|^{2}\right)^{1 / 2} \cos (\vartheta-\delta(\omega)) .
\end{gathered}
$$

Therefore, by (4.4),

$$
\begin{gathered}
\iint_{\mathbb{R}^{2}}\left(|A u-B v|^{2}+|B u+A v|^{2}\right)^{p / 2}|\cos (\vartheta-\delta(\omega))|^{p} \\
=\iint|(A u-B v) \cos \vartheta+(B u+A v) \sin \vartheta|^{p}
\end{gathered}
$$




$$
\leq \sqrt{2}^{p}(p-1)^{p}\|u+i v\|_{p}^{p} .
$$

We integrate with respect to normalized Lebesgue measure $d m(\vartheta)$ as we have already done once before. With $\tau_{p}$ as on page 16, one gets (4.3), which is essentially the same as (1.4), so the proof is complete.

\section{The existence of Bellman function. Proof of Theorem 3}

First note that it suffices to consider the case when $M=N$. For in general, the Bellman function on $\Omega \subset \mathbb{C}^{M} \times \mathbb{C}^{N} \times \mathbb{R} \times \mathbb{R}$ can be defined as the restriction of the Bellman function on the domain in $\mathbb{C}^{\max \{M, N\}} \times \mathbb{C}^{\max \{M, N\}} \times \mathbb{R} \times \mathbb{R}$.

Each interval $I \subset \mathbb{R}$ gives rise to its Haar function $h_{I}$, defined by

$$
h_{I}:=\frac{\chi_{I_{+}}-\chi_{I_{-}}}{|I|^{\frac{1}{2}}},
$$

where $I_{-}$and $I_{+}$denote the left and the right half of the interval $I$ respectively, and $\chi_{E}$ stands for the characteristic function of the set $E$, as usual. Let $\mathcal{D}$ denote the standard family of dyadic intervals on the line. It is a wellknown fact that the set $\left\{h_{I} ; I \in \mathcal{D}\right\}$ forms an orthonormal basis of the space $L^{2}(\mathbb{R})$. Every nice complex-valued function with zero average, continuous and supported on (the interior of) one of the dyadic intervals, say on $J$, can be written as the sum of its Haar series: $f=\Sigma_{I}\left\langle f, h_{I}\right\rangle h_{I}$. The summation goes over $\mathcal{D}_{J}:=\{I \in \mathcal{D} ; I \subseteq J\}$. Consider the operator $T_{\sigma} f=\Sigma_{I} \sigma_{I}\left\langle f, h_{I}\right\rangle h_{I}$, where $\sigma=\left\{\sigma_{I} ; I \in \mathcal{D}_{J}\right\}$ is any sequence of unimodular complex numbers.

We can extend this notions to $\mathbb{C}^{M}$. Define, for $j \in\{1, \ldots, M\}$ and any interval $I \subset \mathbb{R}$, functions $h_{I}^{j}: \mathbb{R} \longrightarrow \mathbb{C}^{M}$ by

$$
\begin{gathered}
h_{I}^{j}=\left(0, \ldots, 0, h_{I}, 0, \ldots, 0\right) . \\
\uparrow \\
j-\text { th place }
\end{gathered}
$$

The set $\left\{h_{I}^{j} ; I \in \mathcal{D}_{J}, 1 \leq j \leq M\right\}$ is an orthonormal basis for the space $L^{2}\left(J \rightarrow \mathbb{C}^{M}\right)$.

Take an arbitrary collection $\sigma=\left\{\sigma_{I}^{j} \in S^{1} ; I \in \mathcal{D}_{J}, 1 \leqslant j \leqslant M\right\}$. For $f \in L^{2}\left(J \rightarrow \mathbb{C}^{M}\right)$ define

$$
T_{\sigma} f=\sum_{\substack{I \in \mathcal{D}_{j} \\ 1 \leqslant j \leqslant M}} \sigma_{I}^{j}\left\langle f, h_{I}^{j}\right\rangle h_{I}^{j} .
$$


The operators $T_{\sigma}$ will be called martingale transforms. Note that $\left\langle f, h_{I}^{j}\right\rangle=$ $\left\langle f_{j}, h_{I}\right\rangle$, where $f=\left(f_{1}, \ldots, f_{M}\right)$. Finally, denote

$$
\left\langle f, h_{I}\right\rangle:=\sum_{j=1}^{M}\left\langle f, h_{I}^{j}\right\rangle h_{I}^{j}=\left[\begin{array}{c}
\left\langle f_{1}, h_{I}\right\rangle \\
\vdots \\
\left\langle f_{M}, h_{I}\right\rangle
\end{array}\right] h_{I} .
$$

We aim to utilize our "model" operator $T_{\sigma}$. The logic will be the following. First, let us find the sharp estimate of $\left\|T_{\sigma}\right\|_{L^{p}(\mathbb{C}) \rightarrow L^{p}(\mathbb{C})}$, i.e. the martingale transform for $M=1$, in terms of $p$. This problem was solved by Burkholder. He found out in [5] that

$$
\sup _{\sigma}\left\|T_{\sigma}\right\|_{p} \leq p-1 .
$$

He proved (5.1) by constructing a function of two real variables (actually another Bellman function) with certain convexity and size properties. The reader is referred to the papers of Burkholder [5], [6] or the book of D. Stroock [18] to study his approach. In particular, on page 344 of [18] it is written about (5.1): "Quite recently Burkholder has discovered the right argument: (...) it is completely elementary. Unfortunately, it is also completely opaque. Indeed, his new argument is nothing but an elementary verification that he has hot the right answer; it gives no hint about how he came to that answer". Further on "for those who want to know the secret behind his proof, Burkholder has written an explanation in his article" [5]. Here is Burkholder's function, again for $p \geq 2$ :

$$
b(x, y)=(|x|-(p-1)|y|)(|x|+|y|)^{p-1} .
$$

Actually stochastic Bellman PDE explains readily the way to write this function, and this is made, for example, in [19].

We want to use this Bellman function of Burkholder in solving our problem, but we are unable to do that. The reason is simple. The variables of the Burkholder's function stand for certain martingales, which in are his case related: one is subordinate to the other. And subordination is in a sense a differential relation "of the first order". In our case we replace these variables not by martingales but by functions: the first is $R_{1}^{2} f$, the second is $f$ (a test 
function). There is no subordination here. The only differential relationship between these two functions (actually between their heat extensions) is

$$
\frac{\partial}{\partial t} \widetilde{R_{1}^{2} f}=-\frac{\partial^{2}}{\partial x_{1}^{2}} \widetilde{f}
$$

This is a differential relation of the second order, and being such it has no connection with convexity of the Burkholder's function. It would be connected to convexity would it be a differential relation of the first order. What we mean can be illustrated by this example. It is obvious that a superposition of convex function $a$ with linear function $l$ is convex, but a superposition of convex $a$ and convex $l$ may not be convex $\left(a(x)=e^{-x}\right.$, $\left.l(x)=x^{2}\right)$. That is exactly the obstacle to use Burkholder's function and compose it with our second order Riesz transforms.

Of course, maybe there is a way around this. But we prefer another approach. It follows the approach in [16].

Idea: we formulate Burkholder's inequality in equivalent (dual) form. The resulting inequality generates another Bellman function. This will be our $B$ from Theorem 3.

We will use the following lemma due to Burkholder.

Lemma 5. Let $(\mathfrak{W}, \mathcal{F}, P)$ be a probability space, $\left\{\mathcal{F}_{n} ; n \in \mathbb{N}\right\}$ a non-decreasing sequence of sub- $\sigma$-algebras of $\mathcal{F}$ and $H$ a separable Hilbert space. Furthermore, let $\left(X_{n}, \mathcal{F}_{n}, P\right)$ and $\left(Y_{n}, \mathcal{F}_{n}, P\right)$ be $H$-valued martingales satisfying

$$
\left\|X_{0}(\omega)\right\|_{H} \leq\left\|Y_{0}(\omega)\right\|_{H}, \quad\left\|X_{n}(\omega)-X_{n-1}(\omega)\right\|_{H} \leq\left\|Y_{n}(\omega)-Y_{n-1}(\omega)\right\|_{H}
$$

for all $n \in \mathbb{N}$ and almost every $\omega \in \mathfrak{W}$. Then for any $p \in(1, \infty)$

$$
\left\|X_{n}\right\|_{L^{p}(P, H)} \leq\left(p^{*}-1\right)\left\|Y_{n}\right\|_{L^{p}(P, H)} .
$$

From the lemma we can easily obtain our next theorem. We will use $\langle f\rangle_{I}$ to denote $\frac{1}{|I|} \int_{I} f(x) d x$.

Theorem 4. Choose $J \in \mathcal{D}$ and let $M \in \mathbb{N}$. Then, for any functions $f \in L^{p}\left(J \rightarrow \mathbb{C}^{M}\right)$ and $g \in L^{q}\left(J \rightarrow \mathbb{C}^{M}\right)$,

$$
\frac{1}{4|J|} \sum_{\substack{I \in \mathcal{D} \\ I \subseteq J}}\left|\langle f\rangle_{I_{+}}-\langle f\rangle_{I_{-}}\right|\left|\langle g\rangle_{I_{+}}-\langle g\rangle_{I_{-}}\right||I| \leq\left(p^{*}-1\right)\left\langle|f|^{p}\right\rangle_{J}^{1 / p}\left\langle|g|^{q}\right\rangle_{J}^{1 / q}
$$


Proof. Take $H=\mathbb{C}^{M}$ and let $\mathcal{F}_{n}$ be $\sigma$-algebra generated by all dyadic subintervals of $J$ with length at least $2^{-n}$. For $\omega \in J$ define

$$
Y_{n}(\omega):=\sum_{\substack{I \in \mathcal{D}_{J} \\|I|>2^{-n}|J|}}\left\langle f, h_{I}\right\rangle h_{I}(\omega) .
$$

Choose a sequence of numbers $\sigma_{I}^{j} \in S^{1}$ and consider

$$
X_{n}(\omega):=\sum_{\substack{I \in \mathcal{D}_{J} \\|I|>2^{-n}|J|}} \sigma_{I}\left\langle f, h_{I}\right\rangle h_{I}(\omega) .
$$

Here $\sigma_{I}$ can be thought of as vectors $\left(\sigma_{I}^{1}, \ldots, \sigma_{I}^{M}\right)$. It follows immediately from the construction that both $\left(X_{n}, \mathcal{F}_{n}, d x\right)$ and $\left(Y_{n}, \mathcal{F}_{n}, d x\right)$ are martingales. Clearly

$$
Y_{n+1}-Y_{n}=\sum_{\substack{I C J \\|I|=2^{-n}|J|}}\left\langle f, h_{I}\right\rangle h_{I}
$$

as well as

$$
X_{n+1}-X_{n}=\sum_{\substack{I I J J \\|I|=2^{-n}|J|}} \sigma_{I}\left\langle f, h_{I}\right\rangle h_{I} .
$$

Since the sums above comprise functions whose supports have disjoint interiors, and $\left|\sigma_{I}^{j}\right|=1$, these martingales satisfy the assumptions of Burkholder's lemma. Now $\|f\|_{L^{p}}=\lim _{n \rightarrow \infty}\left\|Y_{n}\right\|_{L^{p}}$ and $\left\|T_{\sigma} f\right\|_{L^{p}}=\lim _{n \rightarrow \infty}\left\|X_{n}\right\|_{L^{p}}$.

Burkholder's lemma implies

$$
\left\|T_{\sigma} f\right\|_{L^{p}} \leq\left(p^{*}-1\right)\|f\|_{L^{p}}
$$

for any sequence $\sigma$ as above.

Let us reformulate (5.2) as $\left|\left\langle T_{\sigma} f, g\right\rangle\right| \leq\left(p^{*}-1\right)\|f\|_{L^{p}}\|g\|_{L^{q}}$, where $g=$ $\left(g_{1}, \ldots, g_{M}\right) \in L^{q}\left(J \rightarrow \mathbb{C}^{M}\right)$. Definition of $T_{\sigma}$ now implies

$$
\frac{1}{|J|}\left|\sum_{I \in \mathcal{D}_{J}} \sigma_{I}\left\langle f, h_{I}\right\rangle \overline{\left\langle g, h_{I}\right\rangle}\right| \leq\left(p^{*}-1\right)\left\langle|f|^{p}\right\rangle_{J}^{1 / p}\left\langle|g|^{q}\right\rangle_{J}^{1 / q} .
$$

The expression under the sumation sign actually means

$$
\left\langle\left[\begin{array}{c}
\sigma_{I}^{1}\left\langle f_{1}, h_{I}\right\rangle \\
\vdots \\
\sigma_{I}^{M}\left\langle f_{M}, h_{I}\right\rangle
\end{array}\right],\left[\begin{array}{c}
\left\langle g_{1}, h_{I}\right\rangle \\
\vdots \\
\left\langle g_{M}, h_{I}\right\rangle
\end{array}\right]\right\rangle_{\mathbb{C}^{M}} .
$$


By the Cauchy-Schwartz inequality, it can be estimated as

$$
\leq\left\|\left\langle f, h_{I}\right\rangle\right\|_{\mathbb{C}^{M}}\left\|\left\langle g, h_{I}\right\rangle\right\|_{\mathbb{C}^{M}}
$$

Moreover, we can choose the coefficients $\sigma_{I}^{j}$ so that we actually get equality. Finally notice that

$$
\left\langle f, h_{I}\right\rangle=\frac{\sqrt{|I|}}{2}\left(\langle f\rangle_{I_{+}}-\langle f\rangle_{I_{-}}\right)
$$

and the theorem follows.

Remark. When $M=1$, the equality (5.2) is exactly equal to (5.1). But the point of the preceding theorem is that we need (5.2) for arbitrary $M$ in order to prove Theorem 3, as we will see next. This stronger statement was not provided by nor follows from (5.1), so the previous theorem was indeed necessary.

Proof of Theorem 3. Fix $(\zeta, \eta, Z, H) \in \bar{\Omega}$. Consider all integrable functions $f, g: J \rightarrow \mathbb{C}^{M}$ such that $\zeta=\langle f\rangle_{J}, \eta=\langle g\rangle_{J}, Z=\left\langle|f|^{p}\right\rangle_{J}$ and $H=\left\langle|g|^{q}\right\rangle_{J}$ (it is not difficult to construct such functions). Let

$$
B(\zeta, \eta, Z, H):=\frac{1}{4|J|} \sup \sum_{\substack{I \in \mathcal{D} \\ I \subseteq J}}\left|\langle f\rangle_{I_{+}}-\langle f\rangle_{I_{-}}\right|\left|\langle g\rangle_{I_{+}}-\langle g\rangle_{I_{-}}\right||I|,
$$

where the supremum is taken over all such $f, g$.

The supremum above clearly does not depend on the interval $J$. This observation helps to prove the property (2.1).

Indeed, take $f_{ \pm}: J_{ \pm} \rightarrow \mathbb{C}^{M}$ such that $\left\langle f_{ \pm}\right\rangle_{J_{ \pm}}=\zeta_{ \pm}$and $\left\langle\left|f_{ \pm}\right|^{p}\right\rangle_{J_{ \pm}}=Z_{ \pm}$. Define $f: J \rightarrow \mathbb{C}^{M}$ by $f(x):=f_{ \pm}(x)$ if $x \in J_{ \pm}$. In an analogous way we come up with $g$. Write $a=(\zeta, \eta, Z, H):=\frac{a_{+}+a_{-}}{2} \in \bar{\Omega}$.

Note that

- $\langle f\rangle_{I}=\left\langle f_{ \pm}\right\rangle_{I} \quad$ if $\quad I \subseteq J_{ \pm}$

- $\langle f\rangle_{J}=\zeta \quad$ and $\quad\left\langle|f|^{p}\right\rangle_{J}=Z$

and similarly for $g$. 
Now we can write

$$
\begin{aligned}
B(a) \geq & \frac{1}{4|J|} \sum_{\substack{I \in \mathcal{D} \\
I \subseteq J}}\left|\langle f\rangle_{I_{+}}-\langle f\rangle_{I_{-}}\right|\left|\langle g\rangle_{I_{+}}-\langle g\rangle_{I_{-}}\right||I| \\
= & \frac{1}{4|J|}\left(\sum_{\substack{I \in \mathcal{D} \\
I \subseteq J_{+}}}+\sum_{\substack{I \in \mathcal{D} \\
I \subseteq J_{-}}}\right)+\frac{1}{4|J|}\left|\langle f\rangle_{J_{+}}-\langle f\rangle_{J_{-}}\right|\left|\langle g\rangle_{J_{+}}-\langle g\rangle_{J_{-}} \| J\right| \\
= & \frac{1}{4|J|} \sum_{\substack{I \in \mathcal{D} \\
I \subseteq J_{+}}}\left|\left\langle f_{+}\right\rangle_{I_{+}}-\left\langle f_{+}\right\rangle_{I_{-}}\right|\left|\left\langle g_{+}\right\rangle_{I_{+}}-\left\langle g_{+}\right\rangle_{I_{-}}\right||I|+ \\
& +\frac{1}{4|J|} \sum_{\substack{I \in \mathcal{D} \\
I \subseteq J_{-}}}\left|\left\langle f_{-}\right\rangle_{I_{+}}-\left\langle f_{-}\right\rangle_{I_{-}}\right|\left|\left\langle g_{-}\right\rangle_{I_{+}}-\left\langle g_{-}\right\rangle_{I_{-}}\right||I|+ \\
& +\frac{1}{4}\left|\left\langle f_{+}\right\rangle_{J_{+}}-\left\langle f_{-}\right\rangle_{J_{-}}\right|\left|\left\langle g_{+}\right\rangle_{J_{+}}-\left\langle g_{-}\right\rangle_{J_{-}}\right| .
\end{aligned}
$$

At this point we exploit the fact that the definition of $B$ does not depend on the choice of the interval, as mentioned before. In particular, we can replace $J$ by $J_{+}$or $J_{-}$. Having done that, take the supremum of the expressions in the last line over all $f_{+}$and $f_{-}$as above. This process clearly does not affect the free term, which can be rewritten using the definition of $f_{+}$and $f_{-}$. We get exactly

$$
\frac{B\left(a_{+}\right)+B\left(a_{-}\right)}{2}+\frac{1}{4}\left|\zeta_{+}-\zeta_{-}\right|\left|\eta_{+}-\eta_{-}\right|
$$

which proves (2.1).

This establishes the second inequality of the Theorem 3, whereas the first one, i.e. $0 \leq B(\zeta, \eta, Z, H) \leq\left(p^{*}-1\right) Z^{1 / p} H^{1 / q}$, was proved in Theorem 4 .

Finally, fix a compact $K$ and $0<\varepsilon<1$ small enough for $d(K, \partial \Omega) \geq \sqrt{\varepsilon}$. Let $S$ be the standard mollifier $\left[10\right.$, C.4] on $\mathbb{R}^{6} \equiv \mathbb{C} \times \mathbb{C} \times \mathbb{R} \times \mathbb{R}$. Set $S_{\varepsilon}(x)=\frac{1}{\varepsilon^{6}} S\left(\frac{x}{\varepsilon}\right)$ and consider $B_{\varepsilon}=B * S_{\varepsilon}$. This function is smooth on $\Omega_{\varepsilon}:=\{a \in \Omega ; d(a, \partial \Omega)>\varepsilon\}$. The size inequality can deteriorate on $\bar{\Omega}_{\sqrt{\varepsilon}}$ (and thus on $K$ ) at most by factor $1+\sqrt{\varepsilon}$, whereas the concavity inequality (2.1) does not change on $\Omega_{\varepsilon}$, since $\int_{\mathbb{R}^{6}} S_{\varepsilon}(x) d x=1$. Moreover, we can use the Taylor formula to see that (2.1) is equivalent to the inequality 2' from the theorem. 


\section{Addendum}

Here we demonstrate Lemma 3.

Since the assumption and the conclusion of the Lemma are homogenous inequalities with respect to $v$, it is equivalent to prove:

$$
\text { If } E \subset H \text {, then there is } \tau>0 \text { so that } E \subset E_{\tau} \text {, }
$$

where

$$
\begin{aligned}
E=E_{A} & =\left\{v \in \mathbb{R}^{d} ;\langle A v, v\rangle \leq 2\right\} \\
H & =\left\{v \in \mathbb{R}^{d} ; R r \leq 1\right\} \\
E_{\tau} & =\left\{v \in \mathbb{R}^{d} ; \tau R^{2}+\frac{1}{\tau} r^{2} \leq 2\right\} .
\end{aligned}
$$

Note that $E_{\tau} \subset H$ for every $\tau>0$. Since $A$ is a positive matrix, $E$ (more precisely, its boundary) is an ellipsoid. The geometrical shape of other two sets is also clear.

Suppose that we have the proof in case when $k=0$. Now take arbitrary natural numbers $m, n, k$. If $E \subset H$, then $E^{\prime} \subset H^{\prime}$, where $E^{\prime}$ and $H^{\prime}$ are images of $E$ and $H$, respectively, under the orthogonal projection $\mathbb{R}^{d} \rightarrow$ $\mathbb{R}^{m+n} \times\{0\}^{k}$. Since $E^{\prime}$ is again an ellipsoid, by assumption there is $\tau>0$ such that $E^{\prime} \subset E_{\tau}^{\prime}:=\left\{v \in \mathbb{R}^{m+n} \times\{0\}^{k} ; \tau R^{2}+\frac{1}{\tau} r^{2} \leq 2\right\}$. It is clear that this $\tau$ also satisfies $E \subset E_{\tau}$. Hence it is enough to prove the Lemma for $k=0$.

We may also assume that at some point equality is attained in (3.3). This implies that there is $v \in \mathbb{R}^{d}$ for which $R r=1$ and $\langle A v, v\rangle=2$. In other words, $v \in \partial E \cap \partial H$. For $\lambda=\sqrt{R^{-1} r}$ we have $\lambda R=\lambda^{-1} r=1$. The operator $T=\lambda I_{\mathbb{R}^{m}} \otimes \lambda^{-1} I_{\mathbb{R}^{n}}$ leaves $H$ unchanged, whereas it maps $E$ into some other ellipsoid, whose boundary intersects that of $H$ in a point with $R=r=1$. Finally, there are rotations $U_{m} \in S O(m)$ and $U_{n} \in S O(n)$, such that $U_{m} \otimes U_{n}$ maps this intersecting point into $v_{0}:=v_{m} \oplus v_{n}=(1, \underbrace{0, \ldots, 0}_{m-1}) \oplus(1, \underbrace{0, \ldots, 0}_{n-1}) \in$
$\mathbb{R}^{m} \oplus \mathbb{R}^{n}=\mathbb{R}^{d}$.

To summarize, it suffices to solve the case when $k=0$ and $\partial E$ intersects $\partial H$ at $v_{0}$. Since in this case it is obvious that the only admissible $\tau$ is 1 , our task is reduced to proving that $E$ is contained in $E_{1}=\sqrt{2} B^{d}=: B$, i.e. in the closed ball in $\mathbb{R}^{d}$, centered at 0 and with radius $\sqrt{2}$. 
Intersection $\partial H \cap \partial B$ is the "torus" $T=\left\{v \in \mathbb{R}^{d} ; R=r=1\right\}=$ $S^{m-1} \times S^{n-1}$. Let $\mathcal{P}$ be the family of all 2-dimensional planes in $\mathbb{R}^{d}$ which pass through 0 and $v_{0}$. We would like to find a subfamily $\mathcal{P}^{\prime}$ of planes that intersect with $T$ in more than the obvious two points. Take $P \in \mathcal{P}$. There is $u \in v_{0}^{\perp}$ such that $\|u\|^{2}=2$ and $P=\operatorname{Lin}\left\{v_{0}, u\right\}$. Roughly speaking, this establishes a correspondence between $\mathcal{P}$ and a portion of the sphere $\partial B=\sqrt{2} S^{d-1}$. We can write $u=\{a\} \oplus b \oplus\{-a\} \oplus c$ for some $a \in \mathbb{R}$, $b \in \mathbb{R}^{m-1}$ and $c \in \mathbb{R}^{n-1}$. If $p=\lambda v_{0}+\mu u \in P$ is to intersect $T$ in a point, different than $\pm v_{0}$, then we must have

$$
(\lambda+\mu a)^{2}+\|\mu b\|^{2}=(\lambda-\mu a)^{2}+\|\mu c\|^{2}=1
$$

for some $\lambda, \mu \in \mathbb{R}, \mu \neq 0$ (otherwise $p= \pm v_{0}$ ). After adding and subtracting equations and using that $2 a^{2}+\|b\|^{2}+\|c\|^{2}=\|u\|^{2}=2$, one can see that this set of equations is equivalent to

$$
\begin{aligned}
\lambda^{2}+\mu^{2} & =1 \\
4 \lambda a+\mu\left(\|b\|^{2}-\|c\|^{2}\right) & =0
\end{aligned} .
$$

If $a=0$ then we must have $\|b\|=\|c\|$. For $a \neq 0$ the system admits solutions

$$
\begin{aligned}
\lambda^{2} & =\frac{\left(1-a^{2}\right)^{2}-\|b\|^{2}\|c\|^{2}}{\left(1+a^{2}\right)^{2}-\|b\|^{2}\|c\|^{2}} \\
\mu^{2} & =\frac{4 a^{2}}{\left(1+a^{2}\right)^{2}-\|b\|^{2}\|c\|^{2}} .
\end{aligned}
$$

Note that

$$
\|b\|\|c\| \leq \frac{\|b\|^{2}+\|c\|^{2}}{2}=1-a^{2}<1+a^{2},
$$

thus the denominators are always positive. Hence the solution does not exist (i.e. $P$ does not belong to $\mathcal{P}^{\prime}$ ) if and only if

$$
a=0 \wedge\|b\| \neq\|c\| .
$$

This justifies employing identifications

$$
\mathcal{P} \equiv\left\{u=u(a, b, c) \in \partial B ; u \perp v_{0}\right\}
$$

and 


$$
\mathcal{P}^{\prime} \equiv\{u \in \mathcal{P} ; a \neq 0 \vee\|b\|=\|c\|\},
$$

which in principle imply that the set $\mathcal{P}$ ' is "dense" in $\mathcal{P}$.

We would like to show that $E \cap P \subset B \cap P$ or, equivalently, $\partial E \cap P \subset B \cap P$ for all $P \in \mathcal{P}^{\prime}$. Since $\partial E \cap P$ is an ellipse, this simply follows from the fact that $E \subset H$ and that $T \cap P$ contains at least four different points, as has just been shown.

Finally we note that the collection $\mathcal{P}^{\prime}$ is sufficiently large to conclude that $E \subset B$, which had to be proven.

\section{References}

[1] K. Astala: Area distortion of quasiconformal mappings, Acta Math. 173 (1994), 37-60.

[2] A. Baernstein, S. Montgomery-Smith: Some conjectures about integral means of $\partial f$ and $\bar{\partial} f$, Complex Analysis and Differential Equations, Proc. of Wallenberg Symposium in Honor of Matts Essén, Uppsala (1999), ed. Ch. Kiselman.

[3] R. Bañuelos, P. MÉndez-Hernández: Space-Time Brownian Motion and the Beurling-Ahlfors Transform, Indiana Univ. Math. J., to appear.

[4] R. Bañuelos, G. Wang: Sharp inequalities for martingales with applications to the Beurling-Ahlfors and Riesz transforms, Duke Math. J. 80 (1995), no. 3, 575-600.

[5] D. L. Burkholder: Explorations in martingale theory and its applications, Ecole d'Eté de Probabilités de Saint-Flour XIX - 1989, Lecture Notes in Math. 1464, Springer, Berlin (1991), 1-66.

[6] D. L. Burkholder: Boundary value problems and sharp inequalities for martingale transforms, Ann. Prob. 12 (1984), 647-802.

[7] T. Coulhon, X. T. Duong: Riesz transforms for $p>2$, C. R. Acad. Sci. Paris 332, Série I (2001), 1-6. 
[8] T. Coulhon, D. Müller, J. ZienkiewiCz: About Riesz transforms on the Heisenberg groups, Math. Ann. 305 (1996), 369-379.

[9] O. Dragičević, A. Volberg: Bellman function and dimensionless estimates of Riesz transforms, preprint (2003), 1-38.

[10] L. C. Evans: Partial Differential Equations, AMS, 1998.

[11] R. Fefferman, C. Kenig, J. Pipher: The theory of weights and the Dirichlet problem for elliptic equations, Ann. of Math. 134 (1991), 65-124.

[12] T. Iwaniec, G. MARTin: Riesz transforms and related singular integrals, J. reine angew. Math. 473 (1996), 25-57.

[13] N. Kalton, I. Verbitsky: Nonlinear equations and weighted norm inequalities, Trans. Amer. Math. Soc. 351 (1999), no. 9, 3441-3497.

[14] P. E. Kopp: Martingales and stochastic integrals, Cambridge University Press, 1984.

[15] F. Nazarov, A. VolberG: Heating of the Beurling operator and the estimates of its norm, to appear in St. Petersburg Math. J.

[16] S. Petermichl, A. VolberG: Heating of the Beurling operator: weakly quasiregular maps on the plane are quasiregular, Duke Math. J. 112 (2002), no. 2, 281-305.

[17] S. K. Pichorides: On the best values of the constants in the theorems of M. Riesz, Zygmund and Kolmogorov, Studia Math. 44 (1972), 165-179.

[18] D. W. Stroock: Probability Theory, an Analytic View, Cambridge University Press, 1993.

[19] A. Volberg: Bellman approach to some problems in harmonic analysis, Séminaire sur équations aux dérivées partielles, $\mathrm{n}^{\circ} \mathrm{XX}$, Ecole Polytéchnique, Avril 2002. 
Address: Department of Mathematics Michigan State University East Lansing, MI 48824 USA

e-mail: dragicev@msu.edu volberg@math.msu.edu 